Comparing CPAP. pref with CFlexpref we found no significant difference between mean EPAP on CPAP and mean EPAP on CFlex making it unlikely that preference for CFlex was based only on EPAP reduction. In the 23 patients (CPAPpref $=13$, CFlexpref $=10$ ) who had Vt insp measured we derived an index of the flow resistive load to expiration (Table). The CFlexpref group demonstrated a significant fall in this index of expiratory load. The changes in the CPAPref group were more variable. The findings are consistent with CFlex preferers generating and sensing a larger reduction in expiratory load on CFlex.

Abstract P259 Table 1

\begin{tabular}{|c|c|c|c|}
\hline \multicolumn{4}{|c|}{$\begin{array}{l}\text { Resistance to Expiration } \\
\text { (mean EPAP/mean Expiratory Flow) } \\
\text { Geometric mean (SD) } \\
\text { cm He } \mathrm{O} \mathrm{I}^{-1} \mathrm{~s}\end{array}$} \\
\hline \multicolumn{2}{|r|}{ CPAP } & \multicolumn{2}{|c|}{ CFlex } \\
\hline $\begin{array}{l}\text { CPAP Preferred } \\
(n=13)\end{array}$ & $\begin{array}{l}\text { CFlex Preferred } \\
(n=10)\end{array}$ & $\begin{array}{l}\text { CPAP Preferred } \\
(n=13)\end{array}$ & $\begin{array}{l}\text { CFlex preferred } \\
(n=10)\end{array}$ \\
\hline $\begin{array}{l}70.8^{*} \\
(38.3-131)\end{array}$ & $\begin{array}{l}67.6^{* *} \\
(51.6-88)\end{array}$ & $\begin{array}{l}60.25^{*} \\
(34.5-105)\end{array}$ & $\begin{array}{l}54.9^{* *} \\
(40.6-74.3)\end{array}$ \\
\hline
\end{tabular}

Paired t test: ${ }^{*} p \mathrm{NS} ;{ }^{* *} p<0.03 \mathrm{v}$

\section{P260 HAEMATOLOGICAL CHARACTERISTICS OF PATIENTS REFERRED FOR INVESTIGATION OF OBSTRUCTIVE SLEEP APNOEA}

doi:10.1136/thoraxjnl-2012-202678.352

'B Prudon, ${ }^{2}$ W Osborne, 'SD West. 'Newcastle Regional Sleep Service, Newcastle upon Tyne Hospitals NHS Foundation Trust, Newcastle upon Tyne, UK; ${ }^{2}$ Haematology Department, Newcastle upon Tyne Hospitals NHS Foundation Trust, Newcastle upon Tyne, UK

Introduction Obstructive Sleep Apnoea (OSA) is a condition with increasing prevalence. Individuals with severe OSA have significant intermittent nocturnal hypoxia. Erythropoiesis is stimulated through the renal secretion of erythropoietin in response to hypoxia, and is responsible for polycythaemia found in chronic respiratory failure. There are case reports of secondary polycythaemia attributed to OSA, but there are limited data assessing the relationship of OSA physiological parameters and haemoglobin. We aimed to investigate this

Methods Clinical information was collated prospectively from patients assessed at the Newcastle Regional Sleep Service with suspected OSA. None of those included had been referred to investigate polycythaemia. All patients included underwent either a domiciliary or in-patient sleep study as per standard clinical practise, and had a Full Blood Count (FBC) taken.

Results There were 103 patients included: 70\% males, mean (SD) age 52 years (range 26-76 years), BMI $36.1 \mathrm{~kg} / \mathrm{m}^{2}$ (8.3), Epworth Sleepiness Scale 13 (5), daytime $\mathrm{SpO}_{2}$ on air $96 \%$ (2.7) [six patients had $\mathrm{SaO} 2<92 \%$. Sleep study results showed the Apnoea-Hypopnea Index (AHI) to be $<5$ in $13 \%$ [no OSA], $\geq 5$ and $<15$ in $30 \%$ [mild OSA], $\geq 15$ and $<30$ in $19 \%$ [moderate OSA], and $\geq 30$ in $38 \%$ [severe OSA]. Analysis of FBC results showed no statistical difference in Haemoglobin between the four patient groups; mean (SD), no OSA $14.3 \mathrm{~g} / \mathrm{dl}$ (2.1), mild 14.6g/dl (1.5), moderate OSA 14.5g/dl (1.2), severe OSA $14.7 \mathrm{~g} / \mathrm{dl}$ (1.4). There was no correlation between Haemoglobin $(\mathrm{Hb})$ and AHI, oxygen desaturation index (ODI), \% time $\mathrm{SpO}_{2}<90 \%$, \% time $\mathrm{SpO}_{2}<80 \%$, or awake $\mathrm{SpO}_{2}$. There was no significant difference in haematocrit or RBC between groups. A weak positive correlation was observed between total WBC and
ODI, $r=0.319, p=0.001$, but there was no statistical difference between patient groups.

Conclusions In this sleep clinic patient group, we found no correlation between haemoglobin and any OSA severity marker. This suggests that the nocturnal intermittent hypoxia which occurs in OSA alone does not lead to secondary polycythaemia. Further work will evaluate the prevalence of OSA in people with secondary polycythaemia, and whether the OSA is contributory or reflects the general population prevalence of OSA.

\section{P261 ANALYSIS OF THE PREVALENCE AND PREDICTORS OF OBESITY HYPOVENTILATION SYNDROME IN A COHORT OF OVERWEIGHT PATIENTS WITH SUSPECTED SLEEP DISORDERED BREATHING}

doi:10.1136/thoraxjnl-2012-202678.353

V Macavei, K Spurling, J Loft, H Makker. North Middlesex University Hospital, London, UK

Introduction The need for early detection of Obesity Hypoventilation Syndrome (OHS) is clear because delay in the diagnosis and treatment is associated with significant morbidity and mortality.

Objective To determine the prevalence of obesity hypoventilation syndrome among obese patients with suspected sleep apnoea and determine the validity of previously reported predictors of OHS such as serum bicarbonate level.

Methods A retrospective analysis of prospectively collected sleep clinic data on 525 consecutive obese patients referred to sleep clinic from January 2009 to January 2011 to a university hospital was performed. Subjects with suspected sleep disordered breathing were evaluated according to our clinical protocol and capillary blood gases were measured in obese (BMI> 30) subjects.

Results 525 consecutive patients (mean age $51.44 \pm 12.7$, 65.71\% males, mean BMI 34.59 \pm 8.1 ) were evaluated. A total of 344 $(65.52 \%)$ were obese (mean age $52.29 \pm 12.4$, 63.66\% males) of which 128 (37.2\%) were morbidly obese (BMI>40 kg/m²). Daytime hypercapnia $\left(\mathrm{paCO}_{2}>6 \mathrm{kPa}\right)$ was detected in $20.63 \%(71 / 344)$ obese and $22.1 \%(61 / 275)$ obstructive sleep apnoea (OSA) patients. Univariate analysis of potential predictors of OHS showed significant correlations between $\mathrm{paCO}_{2}$ and BMI, FEV1, FVC, AHI, mean nocturnal $\mathrm{SpO}_{2}$, minimum nocturnal $\mathrm{SpO}_{2}$, sleep time spent with $\mathrm{SpO}_{2}<90 \%, \mathrm{paO}_{2}$ and serum $\mathrm{HCO}_{3}$. Following stepwise multiple regression, $\mathrm{paO}_{2}$ and $\mathrm{HCO}_{3}$ were found to be independent predictors of OHS explaining $27.7 \%$ of paCO2 variance $(p<0.0001)$.

On logistic regression analysis, serum $\mathrm{HCO}_{3}$ cut-off of $>27$ mmol was found to have $85 \%$ sensitivity and $90 \%$ specificity for diagnosis of OHS

Conclusion We confirmed high prevalence of OHS in obese patients with OSA (22.1\%) that would be possible to diagnose by measuring serum $\mathrm{HCO}_{3}$ levels, thereby eliminating the need for arterial blood gas sampling.

\section{P262 AGE AND GENDER SPECIFIC DIFFERENCES IN EXCESSIVE DAYTIME SLEEPINESS}

doi:10.1136/thoraxjnl-2012-202678.354

1P Drakatos, ${ }^{2}$ Jarrold, ${ }^{2} \mathrm{~J}$ Harris, ${ }^{2} \mathrm{~A}$ Abidi, ${ }^{3} \mathrm{~A}$ Douiri, ${ }^{1} \mathrm{~N}$ Hart, ${ }^{1} \mathrm{C}$ Kosky, ${ }^{3} \mathrm{~A}$ Williams, 'J Steier. 'Guy'sSt Thomas' NHS Foundation Trust, London, UK; ${ }^{2}$ British Lung Foundation, London, UK; ${ }^{3}$ King's College London, London, UK

Introduction The pictorial Epworth Sleepiness Scale (ESS) (Ghiassi et al., Thorax 2011) has been developed and validated against the traditional ESS and allows subjects to intuitively answer the questions related to daytime sleepiness with pictorial items. Although non-specific, the ESS has been validated for sleep apnoea 